\title{
Uplink-Downlink LTE Multi Cell Capacity: A Performance Analysis in the Presence of ICl, Imperfect Channel Information and Reuse-1 Plan
}

\author{
Belal Abuhaija, IEEE member \\ Sensor Networks and Cellular Systems (SNCS) Research Centre \\ University of Tabuk, Saudia Arabia
}

\begin{abstract}
Long Term Evolution (LTE) technology is based on Orthogonal Frequency Division Multiple Access (OFDMA) technique in the downlink to support multiple users in the same cell at the same time. Such system is known to be susceptible to Inter Cell Interference (ICI) in the downlink. The uplink technology of choice, however, has been SC-FDMA due to low power consumption requirement by the mobile terminal. In order to deliver higher data rates anywhere in the cell in the downlink, especially at the cell edge; many algorithms have been proposed for interference mitigation and avoidance which impose additional complexity on the system and yields minimum capacity enhancements. MIMO techniques have proven to be more efficient than such algorithms. In this paper, a performance analysis of LTE cell capacity is conducted in the presence of several MIMO deployments, where multiple antennas at both the transmitter and the receiver are considered. A comprehensive simulation study of different multiple antenna configurations in the presence of uplink and downlink ICI and cell edge throughput is presented. We also provide insights into the MIMO deployment of choice based on users SINR.
\end{abstract}

\section{Keywords}

LTE, MIMO, MRC, SM, ICI, Rayleigh fading .

\section{INTRODUCTION}

Wireless communication has seen tremendous growth in the past few years driven by the ever-increasing demand for higher data rates and associated computing platforms. LTE and LTE advanced (LTE-A) promised to deliver higher cell capacity and edge user throughput. Both technologies targeted $100 \mathrm{Mbps}$ and beyond with improved spectral efficiency for cell edge customers. The most limited resource of the technologies is the spectrum availability. The deployment of LTE cells with multiple antennas and the density of the deployment can improve on service deployment and the number of customers served. However, this deployment will raise some technical issues such as frequency reuse plan, interference and power consumption. Most of the interference is caused by inter cell interference as opposed to intra cell interference, which when coupled with power has a direct impact on the cell and system capacity.

The uplink technology of choice in LTE has been SC-FDMA due to low power consumption requirement by the mobile terminal; while OFDMA is used in the downlink. In order to deliver higher data rates at the cell edge in the downlink, many algorithms have been proposed for interference mitigation and avoidance. Such algorithms impose additional complexity on the system such as heavy signaling with minimum capacity enhancements.

With the near elimination of intra cell interference through the use of OFDMA in the downlink and SC-FDMA in the uplink within a single cell, by controlling the received power from all users, the presence of inter cell interference probability at the cell edge users can still greatly hinder the cell capacity.

Inter cell interference mitigation relies on scheduling techniques, which aim at reducing the effect of collided resources on SINR and hence improve cell throughput especially for edge users.

Many solutions have been proposed to solve the inter cell interference problem [4, 5, and references therein]. In this paper, LTE physical layer with multiple input single output (MISO), single input multiple output (SIMO) and MIMO configurations are used to enhance SINR in the presence of fading channels and inter cell interference by neighboring cells. However, most ICIC scheduling algorithms proposed in the literature are complex and require heavy signaling measurements and coordination among base stations $[29,37]$ and/or central controller unit [13]. Thus, we are subjecting our simulation studies to random interference which makes the simulation more practical and less complex and, furthermore, yielding more accurate results as shown in [7]. Coupling such random interference with MIMO techniques will provide better insights into the LTE multi cell system performance.

Multiple transmit and receive antennas (MIMO) can be used to enhance signal to interference plus noise ratio (SINR) in cellular systems through diversity and multiplexing gains. In MIMO systems, multipath propagation can be considered as multiple channels between transmitter and receiver. The goal of MIMO technology is to use multiple channels to provide higher total capacity than the theoretical limit for a conventional channel without consuming additional frequency or power and/or to improve the channel quality through enhancing the SINR of the channel. It was shown in [2] that MIMO wireless systems increase the capacity linearly with the minimum number of transmit or receives antennas as compared to single-input singleoutput (SISO) wireless systems.

The rest of the paper is organized as follows. Section II provides a brief literature review while Section III gives a description of the technologies, services, antenna configurations and simulation parameters. In Section IV, the simulator description as well as the simulation environment are presented and illustrated. In Section $\mathrm{V}$, the simulation scenario results are presented and illustrated while Section VI concludes the papers and proposes future work.

\section{LITERATURE REVIEW}

LTE inter cell interference mitigation is a key planning and deployment factor when installing LTE cells. The frequency reuse planning and coordination has been heavily studied by the research community. Inter cell Interference coordination (ICIC) schemes can be of two types, namely static and dynamic. In static ICIC schemes, the basic idea is to control the frequency spectrum used for the cells. Many forms of frequency planning have been studied in the literature which include; frequency reuse-1, reuse3 , fractional frequency reuse (FFR), partial frequency reuse (PFR) and soft frequency reuse (SFR). Frequency reuse planning 
schemes are stemming from one main consideration for traffic load to be stable for the life time of the cell. In reality such an idea is not practical and defies the nature of wireless networks of being adaptive to traffic loads. This is restrictive for resource utilization and system performance [4, 13, 15 and 30]. In [31], a simulation study was presented comparing the reuse-1 scheme with FFR and SFR schemes. The author concluded that the use of reuse- 1 for broadband services can provide better utilization for the system resources when MIMO is used over the static FFR and SFR.

In [5, 6 and 11], it has been reported that the simulation results show that the cell edge users data rates are one third of the inner users (i.e. closer to the cell) when equal transmission power is used for all users in the cell. Adjusting the power ratio between cell edge users and cell center users based on traffic pattern in the cell can yield to better cell performance when using SFR.

Two issues are to be discussed in the context of the above research; First, is that the above studies have been conducted on one transmit and one receive antennas. Second, the traffic patterns are very hard to predict when it comes to broadband services or even constant narrow band services. The traffic load is heterogeneous and significantly changes over time as well as users distribution in the cell.

Many other schemes have been proposed in the literature such as centralized schemes [32-33]. However, building a centralized unit in the infrastructure is not a practical solution as it adds to the latency of the total system, especially since one of LTE design goals is to reduce latency.

Other schemes called semi distributed schemes [34] have divided the problem into two parts; one that is at the central unit where certain resources is allocated to each cell in a super frame while the cell is allocating the resources at a frame level to UE. The overall system is less complex but the need for central entity exists.

Coordinated distributed schemes that are based on information exchange between eNodeB of the allocated RB are the choice for LTE in the presence of X2 interface where nodes can exchange information regarding RB allocations [36]. However, in a very fast fading environment the latency issue should not be ignored or minimized [35]. Other coordinated schemes have partitioned the multi cell optimization problem into a single cell optimization using exchanged messages between serving cells and their corresponding UEs [35, 37].

Most of the above research carries significant complexities and signaling among e-NodeBs. In this paper we are using equal power distribution as proposed in [1], while exploiting MIMO antenna system, allocating resource blocks in order to maximize cell throughput in the uplink and downlink and gauging the outage capacity of cells. Rayleigh fading channel between the transmitter and receiver is employed in the presence of multi transmits / receives antenna system. ICI is accounted for in a random manner as we believe that in heavy traffic loaded system as it is envisioned to be the case in LTE, coordination of cells can impose a huge burden on the system computation and signaling. We used a more subtle technique of random interference generated in random user location.

\section{TECHNOLOGIES AND SERVICES}

\subsection{LTE}

Orthogonal Frequency Division Multiple Access (OFDMA) technology has been the technology of choice for Long Term Evolution (LTE) networks. LTE is envisioned to support data rates up to $300 \mathrm{Mbps}$ in theory and up to $1 \mathrm{Gbps}$. However, the radio resources as in RBs and cell power as well as deployment scenario have a major influence on cells throughput.

In this paper, we propose the utilization of MIMO techniques along with a dynamic resource allocation scheduling algorithm which comprises of PRBs allocation for users and power assignment which maximizes the total cell throughput in the presence of ICI. The impact of inter cell interference in the downlink and uplink on the cell capacity in the presence of random interference from neighbouring cells while no signalling is required or coordination between the cells is measured. The number of customers that are served by each node depends on the number of PRBs utilized in each node. The e arrival process is modelled as Poisson random with inter arrival time of Lambda. The system comprises of 50 PRB each of 12 subcarriers in accordance with LTE standards. We elected to use power allocation first algorithms over the total PRBs as discussed in [1,21].

\subsection{Services}

The services, as per 3G standards, are categorized into four classes; first, conversational class as in speech; second, streaming class which consists of three subcategories: VOIP, video streaming and mobile $\mathrm{TV}$; third, interactive class as in telemetry and games and the fourth is background class as in email services [22]. However, LTE is an all IP packet network; the main services offered are broadband services, even though VoIP is an attractive service, only broadband service is studied in this paper, without users' data rate constraints. The service modeled as streaming $2.5 \mathrm{MB}$ file for downlink, which is a small size streaming file. We consider that if a user is in favorable link conditions then cell data throughput as well as service time is reflected by modulation and coding scheme as illustrated in Table 1 below;

Table 1, Modulation and Coding [7]

\begin{tabular}{|c|c|c|l|}
\hline Mode & Modulation & Code Rate & SINR \\
\hline 0 & 0 & 0 & SINR $<0 \mathrm{~dB}$ \\
\hline 1 & QPSK & .515 & $3.35>$ SINR $=>2.5$ \\
\hline 2 & 16 QAM & .43 & $5.2>$ SINR $=>3.35$ \\
\hline 3 & 16 QAM & .53 & $9.55>$ SINR $=>5.2$ \\
\hline 4 & 64 QAM & .66 & $14.6>$ SINR $=>14.6$ \\
\hline 5 & 64 QAM & .8 & $19>$ SINR $=>14.6$ \\
\hline 6 & 64 QAM & .9 & SINR $>19.0$ \\
\hline
\end{tabular}

\subsection{System Model}

Let us assume that the scheduled radio resource block $\mathrm{SRB}=\{1$, $2,3, \ldots \ldots, r\}$ is $180 \mathrm{kHz}$ for sub frame duration $T s=1 \mathrm{~ms}$. The minimum allocation is one SRB of 12 sub-carriers; each subcarrier can carry 7 signaling elements. A set of code rate is defined as $\mathrm{CR}=\{1,2,3, \ldots, C\}$ while the modulation $M=\{2 ; 4$; 6 . The user data rate for each user is

$R \mathfrak{i}$

$$
\begin{aligned}
& =\frac{C R \dot{\mathfrak{t}}}{T \mathfrak{s}} \log _{2} M \mathfrak{i} \sum_{r=1}^{r} S R B \\
& \text { * } 12 \text { (1) }
\end{aligned}
$$


The total user throughput is then given as the following

$$
\mathrm{U}_{\mathrm{T}}=\sum_{r=0}^{r} R_{i}
$$

where $U_{T}$ is the user throughput. The multi cell system is considering 4 LTE cells with reuse-1 deployment and in such scenario the total system throughput is given by

Cnet

$$
=\sum_{n=1}^{N} \sum_{r=1}^{r} R \dot{\mathrm{i}}
$$

Cnet is the total system capacity in the presence of ICIC and channel fading.

Rayleigh fading channel modeled as a complex Gaussian random process with zero mean and double side unit variances $N$ $(0,1)$.

The received SINR can be given as

$$
\text { SINR }=\frac{P t G t G r}{N_{0} N_{f} L F_{m}}
$$

Where $\mathrm{P} t$ is the transmitted power, $\mathrm{G} t$ is the gain of the transmitter, $\mathrm{Gr}$ is the receiver gain, $\mathrm{Nf}$ is the noise figure,

N0 the spectral noise density, L is the measured path loss between receiver and transmitter as well as other losses (i.e. penetration losses, etc.) and $\mathrm{Fm}$ is the channel fade margin. Inter cell Interference coordination that is imposed by different customers in each cell is randomized and adaptive.

\subsection{Antenna Configuration}

LTE adopted various MIMO technologies including transmit diversity, single user (SU)-MIMO, multiuser (MU)-MIMO, closed-loop rank-1 pre-coding, and dedicated beam-forming [25, 26 and 28]. In SU-MIMO scheme, two or four transmit antennas configuration in the downlink are considered, which supports transmission of multiple spatial layers with up to four layers to a given user equipment. The MU-MIMO scheme allows allocation of different spatial layers to different users in the same timefrequency resource, and is supported in both uplink and downlink. The closed-loop rank-1 pre-coding scheme and transmit diversity are used to improve data coverage utilizing SU-MIMO technology based on the cell-specific common reference signal while introducing a control signal message that has lower overhead. While, Closed-Loop or Open-loop spatial multiplexing is used to increase capacity in a channel with high SINR. The choice between such modes of operations actually depends on timely information the UE can provide to eNodeB. However, in heavy loaded traffic system, the information that is provided to the eNodeB will be obsolete by the time that scheduling is required. The dedicated beam forming scheme is used for data coverage extension when the data demodulation based on dedicated reference signal is supported by the user equipment. In [14], the authors proposed a practical beam forming strategy for Closed-Loop MIMO using a similar algorithm to the dirty paper-paper coding. They claim that the new method achieves the same data rates as of the spatial multiplexing with less complexity and implementation. They assume that the channel state information is known at the transmitter. Since the transmitter knows the interfering signal, it can be cancelled as per the dirty paper code without any power penalty. However, in heavy loaded system the information at the transmitter may be obsolete or heavy signaling needs to take place which degrades system performance.
In this paper, several antenna configurations are realized including single transmit and single receive antenna (SISO), a configuration of one transmit antenna and two receive antennas using maximum ratio combining (MRC) or receive diversity [9], a two transmit antennas and one receive antenna $[19,27]$ or transmit diversity and a configuration of two transmit and two receive antennas which is considered as true MIMO are studied. The results are compared to a system consisting of single transmit and receive antenna Fig. 1 .

Table 2: Simulation Parameters

\begin{tabular}{|l|c|}
\hline Parameter & Model \\
\hline Propagation model & Hata COS231 \\
\hline Thermal noise density & $-174 \mathrm{dBm} / \mathrm{Hz}$ \\
\hline Receiver noise figure & $7 \mathrm{~dB}$ \\
\hline Spectrum Bandwidth & $\begin{array}{c}10 \mathrm{MHz} / 50 \\
\text { Resource block }\end{array}$ \\
\hline Broadband Service & $\begin{array}{c}\text { Streaming } \\
2.5 \mathrm{MByte} \text { file }\end{array}$ \\
\hline Subcarrier spacing & $15 \mathrm{KHz}$ \\
\hline Shadowing Log-Normal & $7 \mathrm{~dB}$ \\
\hline Fast Fading & $\begin{array}{c}\text { Rayleigh } \\
\text { Distribution }\end{array}$ \\
\hline ICIC & $\begin{array}{c}\text { Random and adaptive } \\
\text { as stated in [7] p.296 }\end{array}$ \\
\hline MCS & QPSK, 16QAM, 64QAM \\
\hline Propagation Environment & Dense Urban \\
\hline Inter Cell distance & $500 \mathrm{~m}$ \\
\hline Building Penetration Loss & $12 \mathrm{~dB}$ \\
\hline Number of Cells & 4 \\
\hline Base Station power & $44 \mathrm{dBm}$ \\
\hline UE power & $24 \mathrm{dBm}$ \\
\hline Antenna Configurations & MIMO \\
\hline Antenna Gain & $18 \mathrm{dBi}$ \\
\hline & \\
\hline & SISO, SIMO, MISO and \\
\hline & \\
\hline
\end{tabular}

It is important to note that practical equipment can accommodate up to four antennas and equipping mobile equipment with multiple antennas is still a challenge. Therefore; we are limiting our study and evaluation to a maximum of $2 \times 2$ antennas.

Receive diversity as illustrated in SIMO shown in Fig. 1 is used to improve signal to interference plus noise ratio which influences the integrity of the received data; hence providing quality of service (QoS). One data stream is transmitted from both transmitters and the receiver combines the two signals which is called maximal ratio combining (MRC). MRC can improve the signal quality without additional power or frequency. MRC is a diversity combining in which the received signals are weighted with respect to their SINR and then summed [9]. The resulting SINR yields $\sum$ SINRk for all $\mathrm{N} r$ with $\mathrm{N} r$ being the number of receive antennas [38]. 


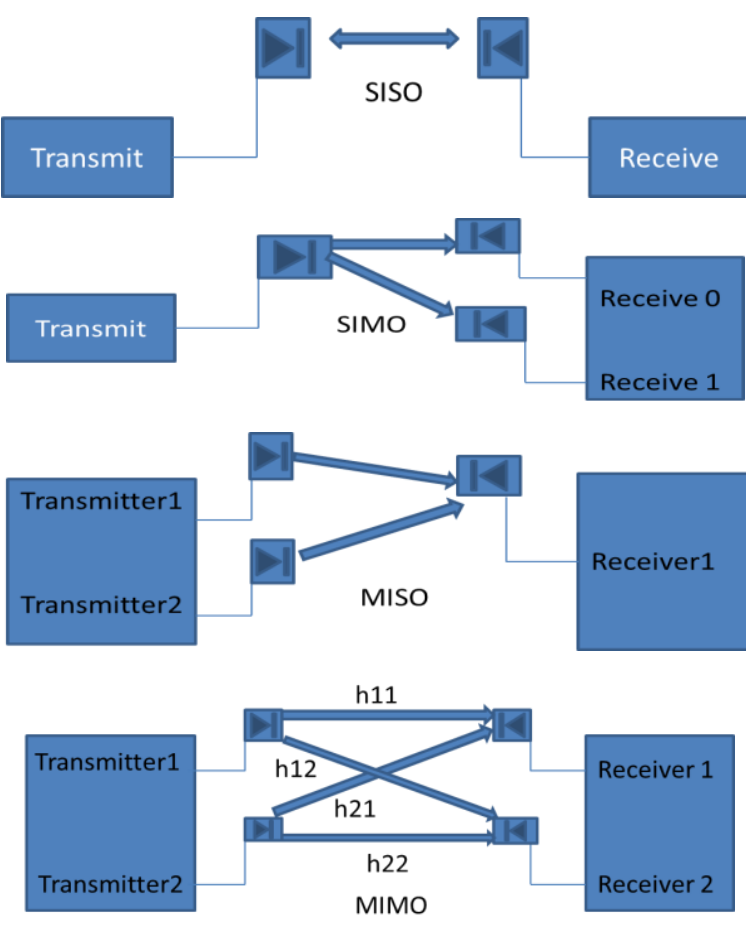

Fig. 1: Antenna Configurations

Transmit diversity as illustrated in MISO shown in Fig. 1 is used to improve signal robustness under fading conditions. The transmitters send the same data but in different parts of the frequency space. The receiver combines the signals which will be higher than if only one transmitter is used (i.e. SISO)

The Alamouti code is designed for two transmit antennas $(\mathrm{Nt}=$ 2 ) and any number of receive antennas. Let $s 1$ and $s 2$ be the transmitted symbols in two consecutive time slots. The transmitted code word is given by

$$
C=\left[\begin{array}{cc}
s 1 & s 2 \\
-s 2^{*} & s 1^{*}
\end{array}\right]
$$

The Alamouti code provides a diversity of $2 \mathrm{Nr}$, and therefore is a full diversity code. In addition, the Alamouti code transmits one symbol per time slot; this is the maximum number of possible symbols for a full diversity code [18]. The detection of the Alamouti code assumes that the channel is quasi-static. The quasi-static assumption is true if the channel did not vary over the Alamouti code word period. Assume that $\mathrm{T}_{\mathrm{c}}$ is the time interval required to transmit each column vector in the matrix $C$. Then, the channel delay spread must be smaller than Tc and the channel coherence time must be larger than 2Tc to be able to decode the Alamouti code word with high probability.

In this paper, the channel matrix for two transmit antennas and single receive antenna can be represented by the corresponding channel gains as follows:

$$
H=\left[\begin{array}{ll}
h 1 & h 2
\end{array}\right]
$$

The transmitted symbols $\hat{\mathrm{S}} 1$ and $\hat{\mathrm{S}} 2$ can be estimated as follows $[19,27]$

$$
\begin{aligned}
& \hat{\mathrm{S}} 1=y_{1} h 1^{*}+y 2^{*} h_{2} \\
& \hat{\mathrm{S}} 2=y_{1} h 2^{*}-y 2^{*} h_{1}
\end{aligned}
$$

where $\mathrm{y} 1$ and $\mathrm{y} 2$ are the received data at for two consecutive time slots and ()* denotes complex conjugate.
Alamouti scheme does not really increase the data rate since we need to transmit $-\mathrm{S}^{*}$ and $\mathrm{Sl}^{*}$ at the second time slot. However, Alamouti scheme shows a 3dB loss compared to MRC.

MISO and SIMO antenna systems achieve SINR gain by combining the signal that takes several paths between transmitters and receivers in a constructive manner. However, there is no really data gain except that comes from improving the SINR and consequently the modulation gain. The capacity gain is logarithmically increasing according to Shannon capacity channel well known formula as in equation (7) below for SISO channel:

$$
C=\log _{2}(1+\operatorname{SINR})
$$

The channel capacity for the SIMO antenna configurations is given by $[7,18,38]$

$\mathrm{C}=\log _{2}(1+N r P / N 0)$

the channel capacity for MISO antenna configurations is given by $[3,7,18,38]$

$$
C=\log _{2}(1+P / N t N 0 W)
$$

Where $\mathrm{P}$ is the total power per $\mathrm{RB}, \mathrm{Nt}$ and $\mathrm{Nr}$ are the number of transmitted and received antennas and $(P / N \mathrm{v} W)$ is the signal to noise power. The channel capacity in the above equations (7), (8) and (9) do not reflect the instantaneous channel conditions between transmitter/s and receiver/s. The channels as illustrated in Fig.3 are modeled as Rayleigh fading channels with independent and identically distributed (i.i.d.) zero-mean complex Gaussian with variance $\sigma^{2}$ at that instant time. The channel matrix $\mathbf{H}$ can be defined as:

$$
H=\left[\begin{array}{ll}
h 11 & h 1 t \\
h r 1 & h r t
\end{array}\right]
$$

Where $r$ and $t$ are the receive antenna and the transmit antenna respectively. Such that the received signal is as in represented in the following:

$$
\hat{\mathrm{S}}=\boldsymbol{H} \mathrm{Y}+\mathrm{N}_{0}
$$

Based on equation (10) and accounting for the channel gain between the receiver and transmitter; the instantaneous capacity of SIMO and MISO respectively shall be:

$$
\begin{aligned}
& \mathrm{C}=\log _{2}\left(1+\sum_{i=0}^{r}\left(|h|^{2} \rho\right)\right) \\
& C=\log _{2}\left(1+\sum_{i=0}^{t}\left(|h|^{2} \rho\right)\right) \\
& \text { where } \rho=N r P / N \mathrm{D} \text { for SIMO and } \rho={ }_{\mathrm{P}} / \mathrm{NtN}_{0} \text { for MISO. }
\end{aligned}
$$

In order to achieve throughput gain where SINR is relatively high; MIMO system technique is used in LTE called spatial multiplexing. Different data streams are transmitted by each antenna which results in an increase in data rate without the need to increase the bandwidth or power. Special multiplexing distributes the total power among the data streams which results in lower power for each stream but since the SINR values are high enough to achieve linear increase in data rates. Spatial multiplexing is the true MIMO system that yields increased data rates. However, the data rates are influenced by ICI as reuse- 1 deployment is used and as illustrated in [7]. 


\section{SIMULATION ENVIRONMENT}

The simulation studies was carried out using a customized version of the Discrete Event simulator as described in $[16,20]$ and as depicted in Fig. 2, with an extension to evaluate cell throughput and service time for broadband services under power constraint. The considered simulation environment is dense urban.

The path loss model used is COST-Hata [23, 27] model for dense urban environment. The simulation parameters used for the downlink are based on the parameters in Table 2.

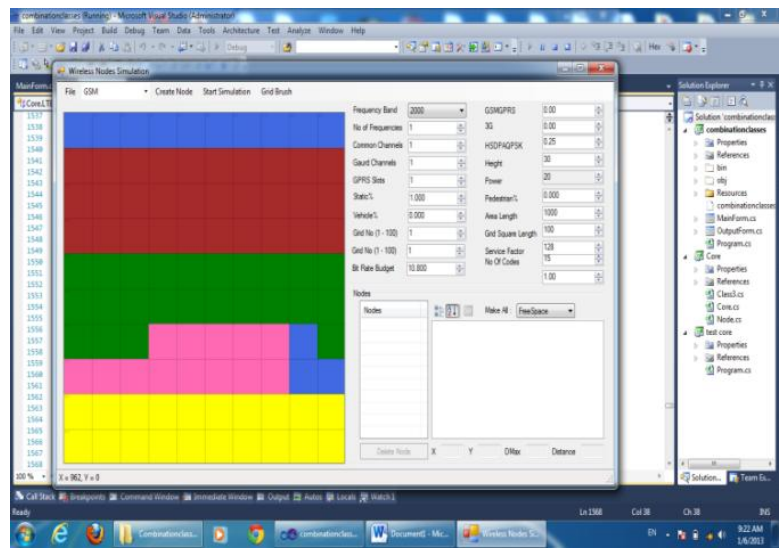

Fig. 2: The Simulator

One service is offered for all users, which is broadband service and all users are assumed to have static mobility in the cell. The simulation scenario is assuming dense urban environment with LTE cell deployment and coverage area of $500 \mathrm{~m}$.

The MCS used in the downlink have three levels of modulations, QPSK, 16QAM and 64QAM where each signalling element carries 2 bits, 4bits and 6bits respectively. MCS values in this paper are based on $[8,10$ and 12].

The customers locations follows a uniform distribution, customers arrival to the system following Poisson process with inter arrival rate of $1 / \lambda$, where $\lambda=10$ as shown in Figure 3.

Cell power is divided equally among all RBs based on the findings of [1 and 17], where water-filling and equal power allocation may yield minor differences. Each customer is assigned 4 RBs while the data rate is influenced by the SINR values and the instantaneous channel conditions which follow Rayleigh distribution.

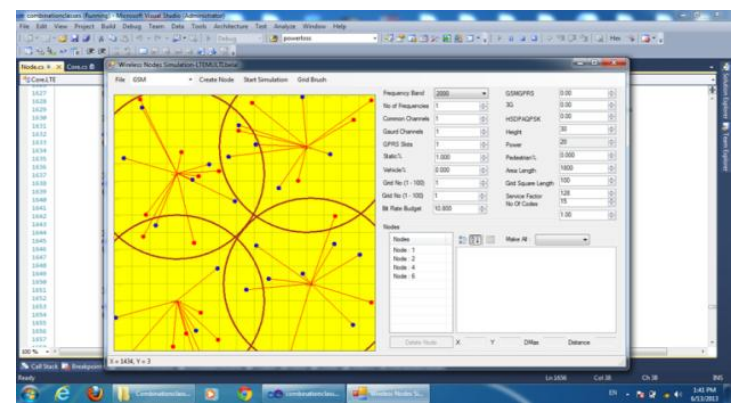

Fig. 3: The Scenario

\section{SIMULATION RESULTS}

The simulation was carried based on the simulation parameters in Table 1 and considering the proposed antenna configurations described in section III. The cell deployment is for macro cell dense urban environment with 500 m radius. The Multi-cell deployment is considered as an outdoor, dense area with no interference coordination among the cells i.e. no resources are assigned for exchanging information. The interference between cells is accounted for by using the studies in [7] and [39] in a randomizing manner. The file transfer size in the downlink is $2.5 \mathrm{MByte}$, while the transmission is assumed to be continuous every time slot with no HARQ retransmissions. It has also been assumed that there is no upper limit on the user's data rate except for the number of assigned PRBs as well as there is no upper limit on the number of users, the users arrival follow Poisson distribution with $\lambda=10$. The considered multiple antenna configurations in this paper emulate a communication scenario where each eNodeB is equipped with two antennas as well as the user equipment (UE). The link level simulator is feeding the system level simulator with the SINR level of each UE. Thus the system level simulator uses such information from the link level simulator to determine the user data rate. Cell edge customers are assigned the same RBs as of the centre edge customers. However, the differentiation between centre edge users and cell edge users is blurred; in the sense that channel condition and interference levels dictate the classifications of users (i.e. centre or edge).

Fig. 4 shows the data rate achieved for UEs based on distance from the base station for downlink and SISO antenna system. As the UEs drift further from the base station the data rate decreases. The modulation and coding is scaled down with SINR value as the power is not used to compensate for the loss of coding or to increase the modulation as it affects the neighbour cells.

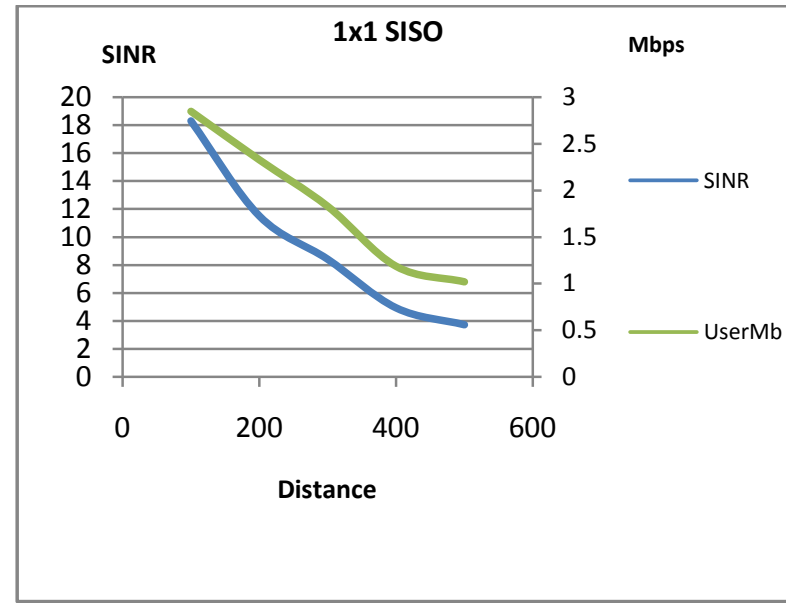

Fig. 4: Downlink SISO Deployment SINR/user data rate

If we are to compare the user throughput based on the assigned number of RBs in this scenario for SISO deployment in the downlink; we notice that the users at $100 \mathrm{~m}$ enjoy almost $3 \mathrm{Mbps}$ while users at $500 \mathrm{~m}$ have a data rate of $1 \mathrm{Mbps}$. The average user throughput is almost 2 Mbps. 


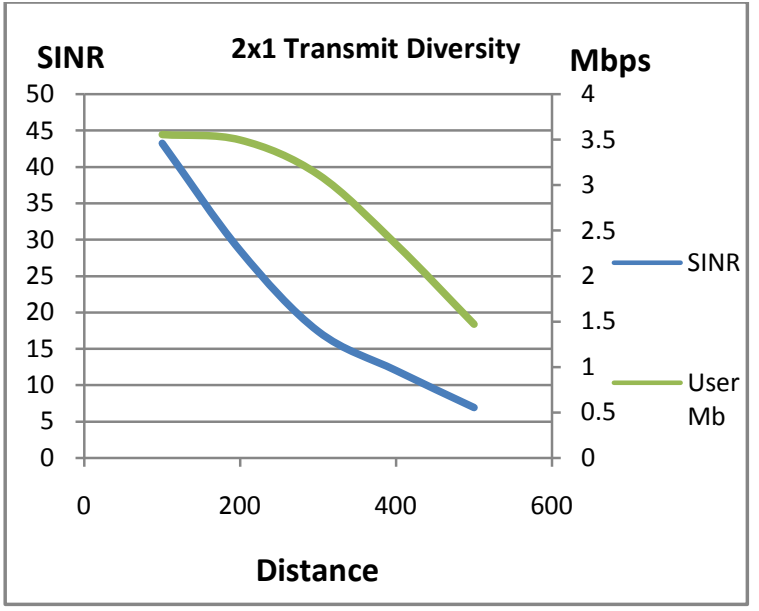

Fig. 5: Downlink Transmit Diversity SINR/User data rate

As illustrated in Figure 5, it is clear that transmit diversity show clear capacity gain over SISO deployment. If we analyse the SINR values it shows that transmit diversity may double the SINR. However, the effect on the cell capacity depends on the large scale fading components and scattering. In other words, when close to the cell centre the benefits of using transmit diversity diminishes when compared to users at the cell edge. This is because the diversity gain enhances the channel capacity according to equation (9). In our simulation we have opted to use Open-Loop transmit diversity as the system is envisioned to be heavy loaded. Minimum feedback information is received by eNodeB. To gauge the effect of inter cell interference effect on the users data rate that is located at the edge of the cells we notice that the collision might reduce the data rate for edge users by $55 \%$ to $65 \%$ especially where customers locations is between $400 \mathrm{~m}-500 \mathrm{~m}$ and depends on the SINR. Transmit diversity as illustrated above can be used to enhance the coverage area and improve signal quality for cell edge users.

For receiver diversity with one transmit antenna and two receive antennas, there are three types of receiver diversity including equal gain combining, selection combining, and maximal ratio combining. Maximal ratio combining receive diversity is illustrated in Figure 6 below

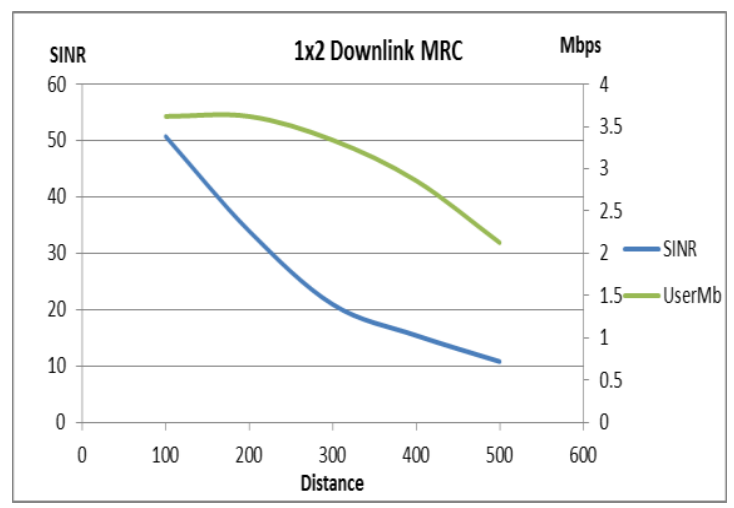

Fig. 6: Downlink MRC 1X2

The receiver diversity as illustrated in Figure 6 shows an increase in data rate with respect to SISO. However, this increase is due to SINR gain which is realised by the multiple copies of the same received signal. The data gain is due to better modulation and coding techniques influenced by higher SINR. Comparing the results in Fig. 5 and Fig. 6, it shows that for limited channel information at the transmitter, MRC has a better capacity gain than transmit diversity. If the channel condition at the transmitter is known then the power split can be adjusted to favour the poor channel and we may get an equal capacity under such assumption. We also noticed that the capacity at the edge of the cells has shown some improvement with transmit diversity and even better capacity with MRC. Therefore, both techniques can be used to enhance coverage and signal quality for edge users. When we introduced the inter cell interference to the cells at the edge we have noticed that the data rate has been decreased by almost $65 \%$ in both antenna configurations.

From the simulation studies, the data rate of the different antenna configurations shows consistency with the capacity equations that have been derived numerically in $[7,18,38]$ for SIMO and MISO even though that we have used a different approach in our simulator. This shows that the simulation results are very close to real life deployment but still needs validation by other operators.

For real gain and true MIMO configuration that influence and even almost double the capacity we just need to add some complexity to the transmitters and receivers by using two transmit antenna and two receive antenna or even using more antennas without any increase in the power or frequency.

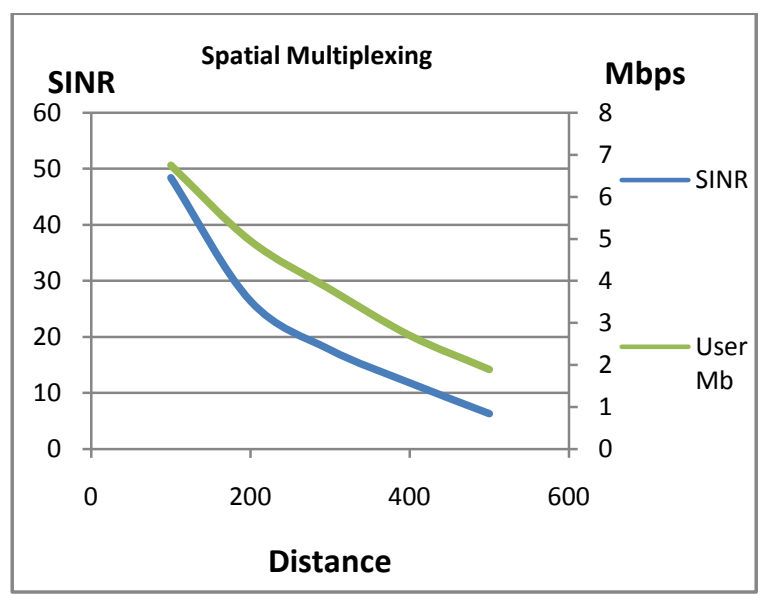

Fig. 7: Spatial Multiplexing Capacity gain

As depicted in Fig. 7, the real capacity gain in the data rates when using MIMO is growing linearly with the number of antennas. The analysis of the results when comparing Fig.5, Fig. 6 and Fig. 7; shows that the capacity grows linearly in Fig. 7 and grows logarithmically in Fig. 5 and Fig. 6. Using the same reasoning for transmit diversity, we use Open-loop spatial multiplexing mode in our simulation. We can conclude that spatial multiplexing is most beneficial for users close to the cell centre and yields marginal capacity gain at the cell edge. Spatial multiplexing may cause more interference at the cell edge with minimal capacity gain.

At the receiver side the data streams that are separated by using interference cancellation algorithms; such as linear zero-forcing, minimum mean squarer-error (MMSE) detector, maximumlikelihood (ML) detector and others [38]. What is very interesting in our findings is that the data rates has shown an increase linearly only when the SINR is greater than $17 \mathrm{~dB}$. If we to compare the results in Fig. 6 and Fig. 7 (i.e. between spatial multiplexing and MRC) we find that the capacity gain if the SINR values is better than a specific value $(17 \mathrm{~dB})$ then MRC will yield better capacity than spatial multiplexing. These findings have inspired us to simulate the scenario where spatial multiplexing combined with MRC as illustrated in Figure 8. 


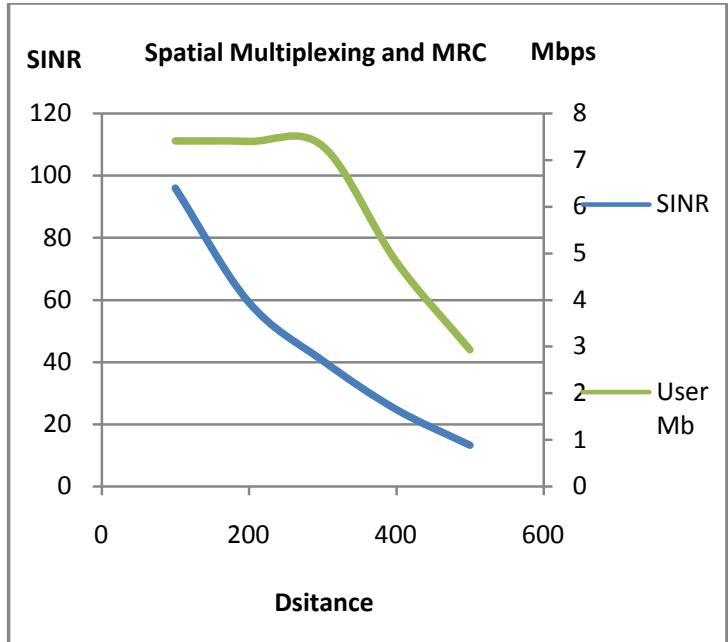

Fig. 8: Spatial Multiplexing Combined with MRC.

When examining the results of combining both Open-Loop spatial multiplexing with MRC we find that the data rates have almost doubled when compared to only MRC and even better spectral efficiency when compared to only spatial multiplexing. However, the inter cell interference has affected the system performance greatly at the cell edge when the SINR values are more than $10 \mathrm{~dB}$. Thus, when deploying the scenario as depicted in Fig.8, we need to account for the power levels at the cells edge which may yield to power reduction.

In the uplink and for SISO configuration, the results as depicted in Figure 9 below:

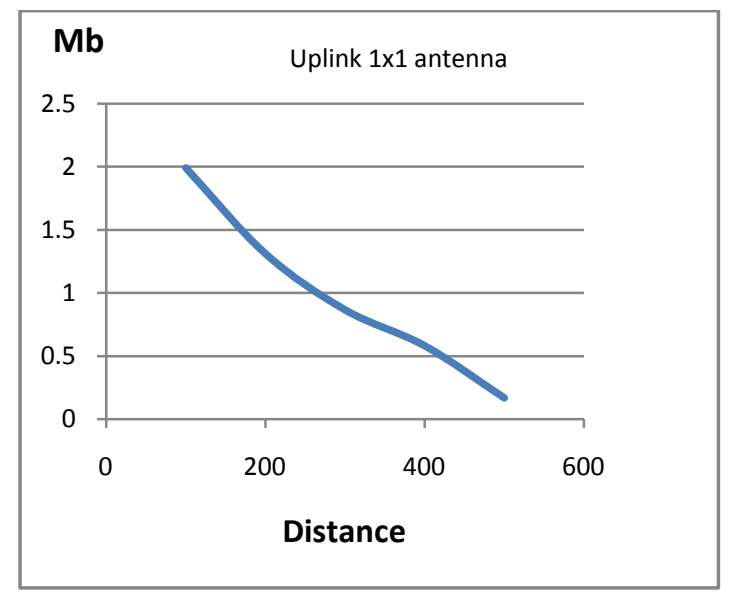

Fig. 9: Uplink SISO

Fig. 10 illustrates the uplink scenario for MRC configuration

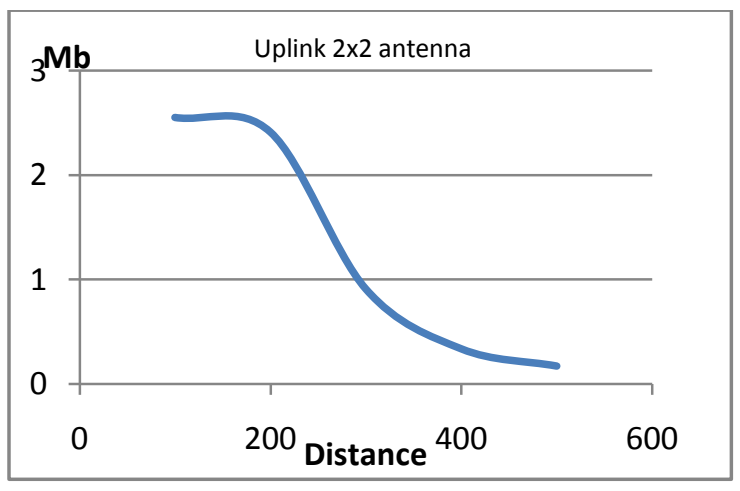

Fig. 10: Uplink MRC
The data rates when using multi antenna in the uplink can improve the spectral efficiency as illustrated from Fig.9 and Fig. 10. However, at distances when the large scale fading components are large enough, the coverage of the cell will shrink below $500 \mathrm{~m}$ when we use $4 \mathrm{RBs}$. Thus we have elected to use only one RB at distances of $400 \mathrm{~m}$ and $500 \mathrm{~m}$. User equipment power and range has to be evaluated and enhanced in a revolutionary matter especially the handheld devices.

\section{CONCLUSION}

In this paper, different multiple antenna systems have been considered for scheduling radio resources in LTE base stations under flat transmit power spectrum. We analyzed Open-Loop modes for different situations under the assumption that the UE in heavy loading conditions will not be able to send timely feedback information to eNodeB. Simple implementation of MIMO techniques under dynamic PRBs allocation for the period of service is provided. The results obtained show that multi antenna techniques can be used to significantly enhance overall system performance. Simulation results were carried out at the system level and in accordance with LTE standards. Inter cell interference is accounted for in a random manner as the randomization on the interference can produce interference diversity gain. From the simulation studies, we noticed that, in terms of enhancing the cell throughput, increasing the power in the form of SINR has limited contribution to data rate increase. However, when more than one antenna used in transmitting and / or receiving, the cells throughputs are enhanced and in some cases have been almost doubled. This is a strong indication that the capacity increases linearly with the number of antennas deployed when using spatial multiplexing and increases logarithmically with the diversities of the transmit and receive. We Conclude that transmit diversity technique is most beneficial for customers with SINR gain around $10 \mathrm{~dB}-12 \mathrm{~dB}$ while spatial multiplexing is more beneficial when SINR gain is around $17 \mathrm{~dB}-18 \mathrm{~dB}$ or more. We also conclude that, with MIMO, we can enhance the system performance as well as the capacity and/or quality of the service signal.

The power optimization and savings need to be evaluated in the presence of a variety of MIMO configurations in the presence of inter cell interference as many studies have concluded that the interference severity increases proportionally with SINR. Therefore future work can be envisioned on designing an algorithm to provide edge users with just enough power for the required service as well as to devise an algorithm that enhances the capacity gain based on prior knowledge of the channel. Work is presently ongoing to address this challenge.

\section{ACKNOWLEDGMENT}

The authors gratefully acknowledge the support for this work by SNCS Research Center at the University of Tabuk under the grant from the Ministry of Higher Education in Saudi Arabia.

\section{REFERENCES}

[1] G. S.V. Hanly, L. Andrew and T. Thanabalasingham, Dynamic allocation of subcarriers and transmit powers in an OFDMA cellular network IEEE Trans. Inform. Theory 55(12) (2009), 5445-5462.

[2] E. Telatar,"Capacity of Multi-Antenna Gaussian Channels", European Transaction on Telecommunication, vol. 10, no. 6, pp:585-595, November 1999.

[3] IST-4-027756 WINNER II, "D3.4.1, The WINNER II Air Interface: Refined Spatial Temporal Processing Solutions," Retrieved Mar. 08, 2010, from https://www.istwinner.org/WINNER2-Deliverables/. 
[4] G. Fodor, "Performance analysis of a reuse partitioning technique for ofdm based evolved utra," in 14th IEEE International Workshop on Quality of Service, 2006. IWQoS 2006, pp. 112-120.

[5] T. Bonald, S. Borst, and A. Proutiere, "Inter-cell scheduling in wireless data networks," in Wireless Conference 2005 Next Generation Wireless and Mobile Communications and Services (European Wireless), 11th European, VDE, 2005, pp. 1-7.

[6] 3GPP R1-050629,“'Inter-cell interference mitigation,'” Huawei, 2005. Available (July2012):http://www.3gpp.org/ftp/tsg ran/wg1 rl1/TSGR1 AH/LTE AH June-05/Docs/R1-050629.zip.

[7] S. Sesia, I. Toufik, and M. Baker, "Lte-the umts long term evolution," From Theory to Practice, published in, vol. 66, 2009.

[8] H. Holma and A. Toskala, LTE for UMTS-OFDMA and SC-FDMA based radio access. Wiley, 2009.

[9] Guan, Zhang-Jun; Zhang, Wei-Jiong; Zhou, Xi-Lang, "Performance analysis of multi-antenna relay communication systems with MRC," International Journal of Communication Systems, vol. 25, no. 11, pp. 15051512,Feb 2012.

[10] 3rd Generation Partnership Project (3GPP), Evolved Universal Terrestrial Radio Access (E-UTRA); Physical Channels and Modulation (Release 11), 3GPP TS 36.211 V11.1.0 (2012-1GPP Std., Dec. 2012.

[11] Abdelbaset S. Hamza,et al, A Survey on Inter-Cell Interference Coordination Techniques in OFDMA-Based Cellular Networks. Communications Surveys \& Tutorials, IEEE (Volume:PP, Issue: 99 ) 07 March 2013.

[12] Alexiou, Antonios; Bouras, Christos; Kokkinos, Vasileios; Papazois, Andreas; Tsichritzis, George, "Modulation and coding scheme selection in multimedia broadcast over a single frequency network-enabled long-term evolution networks", International Journal of Communication Systems, vol. 25, no. 12, pp. 1603-1619,Dec 2012.

[13] Rahman, M. Enhancing cell-edge performance: a downlink dynamic interference avoidance scheme with inter-cell coordination, Wireless Communications, IEEE Transactions on (Volume:9, Issue: 4 ) April 2010.

[14] Sunghyun Cho, Insoo Hwang, Vahid Tarokh, Cheolwoo You" A practical transmit beamforming strategy for closedloop MIMO communication" International Journal of Communication Systems, Volume 25, Issue 8, pages 10911099, August 2012

[15] Qian, Manli ; Hardjawana, Wibowo ; Li, Yonghui ; Vucetic, Branka ; Shi, Jinglin ; Yang, Xuezhi: Inter-cell interference coordination through adaptive soft frequency reuse in LTE networks. WCNC : IEEE, 2012. - ISBN 9781-4673-0436-8, S. 1618-1623.

[16] Abuhaija, B., "Performance analysis of LTE multiuser flat downlink power spectrum and radio resources scheduling", Journal of High Speed Networks, vol.18, no.3,pp: 173-184, 2012.

[17] J. Jang and K. Lee, "Transmit power adaptation for multiuser ofdm systems,"Selected Areas in Communications, IEEE Journal on, vol. 21, no. 2, pp. 171178, 2003.
[18] S. M. Alamouti, "A Simple Transmit Diversity Technique for Wireless Communications," IEEE Journal on Selected Areas in Communications, vol. 16, no. 8, pp. 1451-1458, Oct. 1998

[19] V. Tarokh, H. Jafarkhani, and A. Calderbank, "Space-time Block Codes from Orthogonal Designs," IEEE Transactions on Information Theory, vol. 45, no. 5, pp 1456-1467, Jul. 1999..

[20] B. Abuhaija and K. Al-Begain, "Enhanced common radio resources managements algorithm in heterogeneous cellular networks," in Third International Conference on Next Generation Mobile Applications, Services and Technologies, 2009. NGMAST'09, pp. 335-342.

[21] T. Thanabalasingham, S. Hanly, L. Andrew, and J. Papandriopoulos, "Joint allocation of subcarriers and transmit powers in a multiuser ofdm cellular network," in IEEE International Conference on Communications, ICCO6, IEEE, 2006, pp. 269-274 .

[22] 3rd Generation Partnership Project (3GPP), Technical Specification Group Services and System Aspects; Service Aspects, Service and Service Capabilities, 3GPP TS 22.105 V6.2.0 (2003-06), 3GPP Std., Jun. 2003.

[23] J. Parsons and P. Parsons, The mobile radio propagation channel. Wiley Chichester, UK, 2000, vol. 2.

[24] Mousa, Allam; Mahmoud, Hala, "Reducing ICI effect in OFDM system using low-complexity Kalman filter based on comb-type pilots arrangement," International Journal of Communication Systems, vol. 24, Issue 1, pp.53-61, 2011.

[25] S. Shim, J. Kwak, R. Heath, and J. Andrews, "Block Diagonalization for Multi-User MIMO with Other-Cell Interference," Wireless Communications, IEEE Transactions on, pp. $2671-2681$, Jul. 2008.

[26] V. Tarokh, H. Jafarkhani, and A. R. Calderbank, "Spacetime block coding for wireless communications: performance results," IEEE Journal on Selected Areas in Communications, vol. 17, no. 3, pp. 451-460, Mar. 1999.

[27] J. G. Proakis, Digital Communications. McGraw-Hill, 1995.

[28] 3rd Generation Partnership Project (3GPP), Technical Specification Group Radio Access Network, Spatial Channel Model for Multiple-Input Multiple Output (MIMO) simulations (Release 11), 3GPP TR 25.996 V11.0.0 (201209), 3GPP Std.

[29] 3rd Generation Partnership Project (3GPP), Technica Specification Group Radio Access Network, Evolved Universal Terrestrial Radio Access Network (EUTRAN);X2 application protocol (X2AP) (Release 11), 3GPP TS 36.423 V11.5.0 (2013-06)

[30] N. Hassan and M. Assaad, "Optimal Fractional Frequency Reuse (FFR) and resource allocation in multiuser OFDMA system," in Proc. Int. Conf. Information and Communication Technologies ICICT09., 2009, pp. 88-92.

[31] G. Fodor, Ch. Koutsimanis, A. Rcz, N. Reider, A. Simonsson, and W.Mller, "Inter cell Interference Coordination in OFDMA Networks and in the 3GPP Long Term Evolution System," Journal of Communications,vol. 4, no. 7, pp. 445-453, 2009.

[32] M. Rahman and H. Yanikomeroglu, "Multicell Downlink OFDM Subchannel Allocations Using Dynamic Inter cell 
Coordination," in Proc. IEEE Global Telecommunications Conf. GLOBECOM '07,2007, pp.5220-5225.

[33] M.C. Necker, "A Novel Algorithm for Distributed Dynamic Interference Coordination in Cellular Networks," in Proc. KiVS, pp. 233-238, 2011.

[34] V. Corvino, D. Gesbert and R. Verdone, "A Novel Distributed Interference Mitigation Technique using Power Planning," in Proc. IEEE Wireless Communications and Networking Conf. WCNC 2009, 2009, pp.1-6.

[35] S. Cicalo, V. Tralli, and A.I. Perez-Neira, "Centralized vs Distributed Resource Allocation in Multi-Cell OFDMA Systems," in IEEE $73^{\text {rd }}$ Vehicular Technology Conference, 2011, pp. 1 - 6 .

[36] D. Kimura, Y. Harada, and H. Seki, "De-Centralized Dynamic ICIC Using X2 Interfaces for Downlink LTE Systems," in Proc. IEEE $73^{\text {rd }}$ Vehicular Technology Conf. (VTC Spring), 2011, pp. 1-5.
[37] 3rd Generation Partnership Project; Technical Specification Group Radio Access Network; Evolved Universal Terrestrial Radio Access Network(E-UTRAN);X2 signalling transport (Release 11) 3GPP TS 36.422 V11.0.0 (2012-09).

[38] Jyoti Gangane, Mari Carmen Aguayo-Torres, Juan J.Sanchez, Sharad Wagh4; Maximal Ratio Combining SCFDMA Performance over Land Mobile Satellite Rice fading Channel; International Journal of Emerging Technology and Advanced Engineering, (ISSN 2250-2459, Volume 2, Issue 11, November 2012)

[39] R. Bosisio and U. Spagnolini, "Interference Coordination vs. Interference Randomization in Multi-cell 3GPPLTE System," in Proc. of IEEE Wireless Communications and Networking Conf. (WCNC'08), March 2008. 